\title{
HISTOPATHOLOGICAL EVALUATION OF EFFECT OF LASER VERSUS FOSFOMYSINE IN TREATMENT OF OSTEOMYELITIS INDUCED BY METHICILLIN-RESISTANT STAPHYLOCOCCUS AUREUS (MRSA) IN EXPERIMENTAL ANIMALS
}

\author{
Walaa Samir Abdel-Fatah*
}

\begin{abstract}
Introduction: Fosfomycin has been shown highly effective in the treatment of experimental MRSA osteomyelitis. Laser therapy appeared to be a promising alternative technique to pharmacological agents in the treatment of osteomyelitis. Consequently, this study aimed to determine the effects of fosomycin and laser in the treatment of induced osteomylitis by MRSA in rabbits.
\end{abstract}

Material and methods: Localized osteomyelitis was induced in rabbits by percutaneous injection of $0.1 \mathrm{ml}$ of $10^{8} \mathrm{cfu}$ of MRSA clinical isolate into the intramedullary cavity of tibia. After two weeks, tibial osteomyelitis was confirmed radiographically. Rabbits were divided into three groups (each group contained 10 rabbits): untreated controls group, group treated with fosfomycin $75 \mathrm{mg} / \mathrm{kg}$ subcutaneously every $24 \mathrm{~h}$ and group treated with Diode laser $940 \mathrm{~nm}$, three times weekly. After 14 days of treatment, rabbits were left untreated for 24 hours then euthanized and the tibias harvested. evaluation of treatment was done by measurement of bone density radiologically, Histopathological examination and each tibia were cultured for bacterial counts determination

Results: Bacterial cultures from bones were positive for MRSA in 8 out of $10(80 \%)$ rabbits in the control group, in 9 out of $10(90 \%)$ rabbits in the fosfomycin and laser treated group . Laser group showed the highest bone density among the studied groups. No significant difference was found in the bone cultures and bone density between the three groups; P value 0.302 and 0.157 respectively

KEY WORDS: Osteomyelitis, MRSA, Fosfomycin and laser

* Lecturer of Oral Maxillofacial Radiology; Faculty of Dentistry, Bani-seuif University. 


\section{INTRODUCTION}

Osteomyelitis is an acute or chronic inflammation of the bone caused by an infecting organism (Maynor et al.,1998). Osteomyelitis was classified into three groups: hematogenous osteomyelitis occurring after bacteremia, contiguous osteomyelitis, and osteomyelitis associated with peripheral vascular disease (Waldvogel et al.,1970). Bone is normally resistant to bacterial colonization but events like trauma, surgery, the presence of foreign bodies, or the placement of prostheses may disrupt bony integrity and lead to the onset of bone infection (Brady et al.,2006).

The most commonly involved pathogen is Staphylococcus aureus (S. aureus). MethicillinresistantS.aureus (MRSA) has alsobeen increasingly isolated from chronic osteomyelitis lesions. Other causative pathogens include Staphylococcus epidermidis, Pseudomonas aeruginosa, Serratia marcescens and Escherichia coli. Mycobacterial and fungal infections are generally uncommon and are often associated with immunodeficiency (Conterno and Turchi .2013)

Vancomycin, a glycopeptide in clinical use for more than 50 years and still serves as the cornerstone of the treatment of MRSA ( Howden et al., 2010). However, there are increasing numbers of reports on emergence of vancomycin intermediate sensitive S. aureus (VISA) and vancomycin-resistant S. aureus (VRSA) (Amatya et al., 2014) .In addition, many researchers have reported the higher rates of likelihood of treatment failure leading to higher rates of mortality, due to the infection caused by MRSA having MIC of vancomycin at the upper end of susceptible range (Chen et al., 2014), so an urgent need for alternative antibiotics.

Fosfomycin (FOF) is a well-tolerated broadspectrum bactericidal agent with longstanding sensible clinical use (Popovic et al., 2010). FOF shows antibacterial activity against various Gram- positive and Gram-negative bacteria, including multidrug-resistant strains such as MRSA (Falagas et al., 2008). In addition, high antibacterial activity in biofilms and the enhancement of efficacies of other agents when combined are attributed to fosfomycin (Tang et al., 2013).

It penetrates well into osseous tissue and has proved to be clinically useful in the treatment of acute and chronic osteomyelitis (Roussos et al., 2009). FOF was reported to be highly effective in the treatment of experimental MRSA osteomyelitis in vitro and in vivo (Poeppl et al .,2011).

Laser is the acronym of the words 'Light Amplification by Stimulated Emission of Radiation'. Laser since Albert Einstein described the theory of stimulated emission in 1917 , lasers technology has and is influencing our life in many ways. Its advancements in the field of medicine and dentistry are playing a major role in patient care and wellbeing. (Thomas etal;.1993) . Light therapy has been proposed as an effective treatment for a variety of human conditions. Laser phototherapy, with the appropriate irradiation parameters; appears to be a promising adjunct and/or alternative technique to pharmacological agents in the treatment of osteomyelitis. (Kaya et al; 2010). Laser therapy plays an important role in healing by inhibiting the development of bacteria in infected wounds, and both in vivo and in vitro studies have demonstrated that light at a wavelength of between 405 and 880 $\mathrm{nm}$ has a bactericidal effect on $\mathrm{S}$. aureus. The increasing use over the past 15 years of GalliumAluminum-Arsenide (GaAlAs) diode lasers, which have a higher depth of penetration than other types of lights used in light therapy, offers the clinician a penetrative tool of great efficiency. (Kaya et al; 2010)

The action of lasers on hard and soft tissue as well as bacteria depend on the absorption of laser tissue by chromphore (water, apatite minerals, and various pigmented substances) within the target 
tissue. Better absorption allows for a more efficient photo-thermal sterilization.(Thomasetal,;1993)

This study aimed to determine the effects of fosomycin and laser in the treatment of induced osteomylitis by MRSA in rabbits.

\section{MATERIALS AND METHODS}

Animals: The study was done in compliance with national and international standards for the protection of animals used for scientific purposes, and the project was approved by the ethics committee of the Faculty of Veterinary, Cairo University, Egypt. The experiment was conducted at the Faculty of Veterinary, Cairo University, Egypt. The rabbit mean age at the beginning of the experiment was 7 months and average individual weight was $1500 \mathrm{~g}$. Rabbits were maintained in a temperature-controlled accommodation (16-21 $\left.{ }^{\mathrm{Co}}\right)$. The food and water were provided throughout the experiment period. Accommodation was done in specific cages, 2-3 rabbits in cage.

Bacterial strain: The clinical MRSA strain used in the study was obtained from patients having bacterima undergoing treatment at Egypt Children Hospital for health insurance. The strain was vancomycin intermediate resistant with minimum inhibitory concentration $(\mathrm{MIC})=2 \mathrm{mg} / \mathrm{L}$ determined with E test.

In vitro susceptibility testing of Fosfomycin: MIC of fosfomycin for MRSA strain was determined by agar dilution supplemented with Glucose6-phosphate ( $25 \mathrm{mg} / \mathrm{L})$, according to the recommendations of the Clinical and Laboratory Standards Institute (CLSI 2014) and result were interpreted according to European committee on antimicrobial susceptibility testing criteria (susceptible, $\leq 32$; resistant, $\geq 64 \mathrm{mg} / \mathrm{L}$ ) (EUCAST, 2013).

Induction of osteomyelitis infection: Each rabbit was anesthetized with ketamine and xylazine, and the left hind leg was shaved and disinfected with polyvinyl pyrrolidine-iodine. An 18-gauge needle was inserted percutaneously through the lateral aspect of the left tibial metaphysis into the intramedullary cavity then $0.1 \mathrm{ml}$ of $\operatorname{MRSA}\left(7 \times 10^{8}\right.$ CFU) was injected

After 2 weeks as an incubation period, osteomyelitis of the tibia was confirmed radiographically (Figure 1)

Treatment groups: Thirty rabbits were used in this study and divided into THREE groups as follows:

Group 1: (control group, $\mathrm{n}=10$ ) was infected but left untreated for the duration of the study.

Group 2: (antibiotic group, $\mathrm{n}=10$ ) rabbits were treated for 2 weeks with fosfomycin .

Group 3: $(\mathrm{n}=10)$ rabbits were treated for 2 weeks with Diode laser $940 \mathrm{~nm}$.

Antibiotic preparation: Fosfomycin (FOF) powder was dissolved in sterile water and administered intraperitoneally at a dose of $75 \mathrm{mg} / \mathrm{kg}$ once daily.

Laser irradiation: Deep tissue hand piece of Biolase diode laser $940 \mathrm{~nm} \pm 10 \mathrm{~nm}$ used to administrate 4 watt 480 joul for 120 second to induce biomodulation effect for two weeks 3time in each week at site of injection infection (Fig .2).

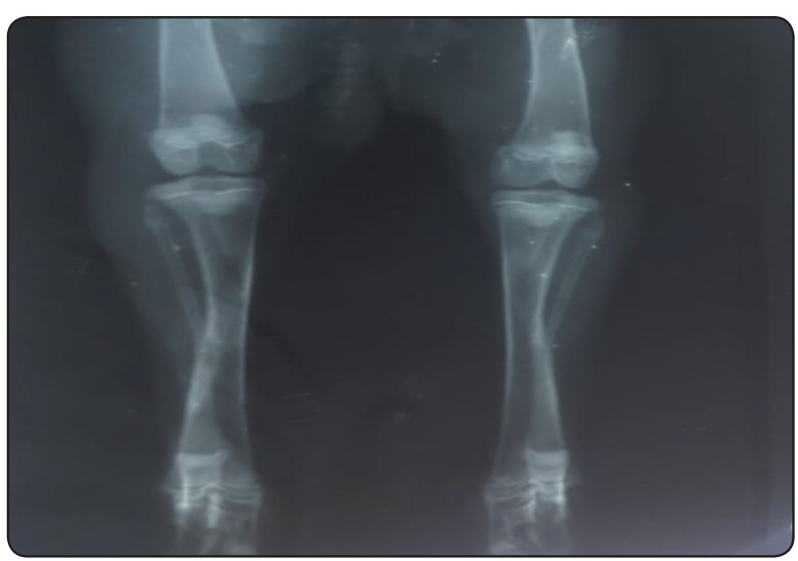

Fig. (1) X -ray of rabbit left and right tibia after 14 days of osteomyelitis induction showed periosteal reaction, area of cortical sclerosis and ill-defined osteolytic lesion of left tibia in comparison to right tibia. 


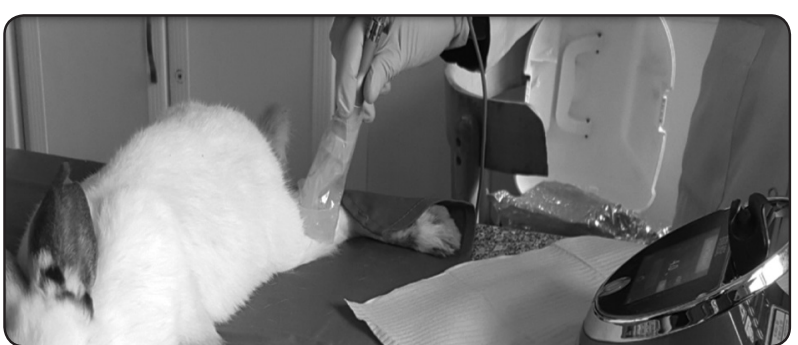

Fig. (2) laser application to rabbit tibia

\section{Bone cultures and bacterial counting:}

Rabbits were sacrificed 24 hours after the completion of antimicrobial and laser treatment; both tibias were removed aseptically, dissected free of all soft tissue, and processed for bacterial cultures. The proximal and distal ends of the tibia were swabbed and streaked onto blood agar plates to check for any bacterial growth then placed into tubes containing $5 \mathrm{~mL}$ of sterile trypticase soy broth. Plates and tubes were incubated at $37^{\circ} \mathrm{C}$ for $24 \mathrm{~h}$ and growth and/or turbidity recorded.

\section{Radiographic evaluation:}

Digital radiographic monitoring was done by examination of both tibias of the rabbits immediately after sacrificed; using a digital image capture device photo-stimulable phosphor plate. The special software of the Digora system (Orion Corporation, Soredex, Medical system, Helsinki, Finland) was used to assess the relative radiographic bone density at the Faculty of Dentistry, Cairo University . Data was collected for all groups and statistically analyzed. Measurements were done at different time intervals by three radiologists, independently of each other (figure 2) the X-Rays were directed perpendicular to specimen and plate. The central ray was directed at 90 degrees to the film which was placed parallel to the tibia using a special film holder (Rinn XCP-film holders, Rinn CO., USA) which was connected to an X-ray machine by an extension arm and a ring to fix the target-film distance at $75 \mathrm{~cm}$ during all the follow-up periods throughout

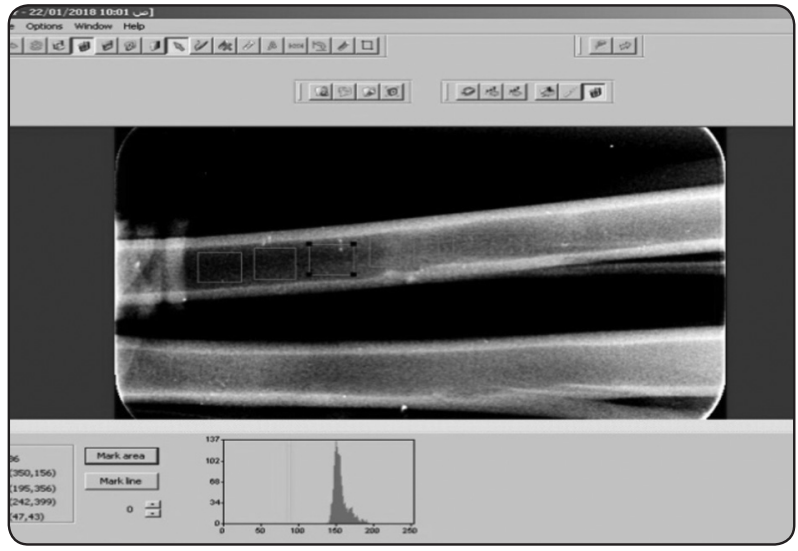

Fig. (3) Radiographic relative bone density measurement

the study. Exposure parameters were kept constant throughout the imaging period.

\section{Histopathological examination:}

\section{Specimen Preparation and Histo-pathologic Ex- amination:}

Histological procedures aimed to provide good quality sections that can be used for a light microscopic evaluation of human or animal tissue changes in either spontaneous or induced diseases. according to Lilhe and Fulmer, 1976 tissues were fixed with neutral formalin $10 \%$, embedded in paraffin, and then manually sectioned with a microtome to obtain 4-5 $\mu$ m-thick paraffin sections that stained with hematoxylin and eosin (H\&E) as following:

i- Block samples of the tibia at the sites injection of infection as well as adjacent bone tissues were dissected and resected, and then fixed in $10 \%$ neutral buffered formalin solution for 2 days.

ii- After fixation, the tissues were demineralized and embedded in paraffin. Then the biopsies were processed for ground sectioning. Consecutive sections $6 \mathrm{~mm}$ thick were cut with a microtome longitudinally in a bucco-lingual plane and parallel to the axis of the central part of the tibia.

iii-The fixed specimens (control and experimental samples) were decalcified in $20 \%$ 
ethylenediamine- tetra-acetic acid (EDTA) PH 7 with a change per week for ten weeks until decalcification was completed. Neutral EDTA decalcifying solution consisting of : EDTA (disodium salt) $26 \mathrm{gm}$, distilled water $1750 \mathrm{~cm}^{3}$ and $26 \mathrm{gm}$ sodium hydroxide was added gradually to adjust the $\mathrm{PH}$ of the solution .

iv- The specimens were dehydrated in ascending grades of ethanol, infiltrated in xylene, embedded in paraffin blocks which were sectioned at a $5 \mu \mathrm{m}$ thickness and stained heamatoxylin and eosin stain (H.\&E.) by the standard technique (Lilhe and Fulmer 1976) .

$\mathrm{v}$-The histological examination was performed with a sterio-microscope (100 X magnification). (fig. 4) (Walaa et al; 2011)

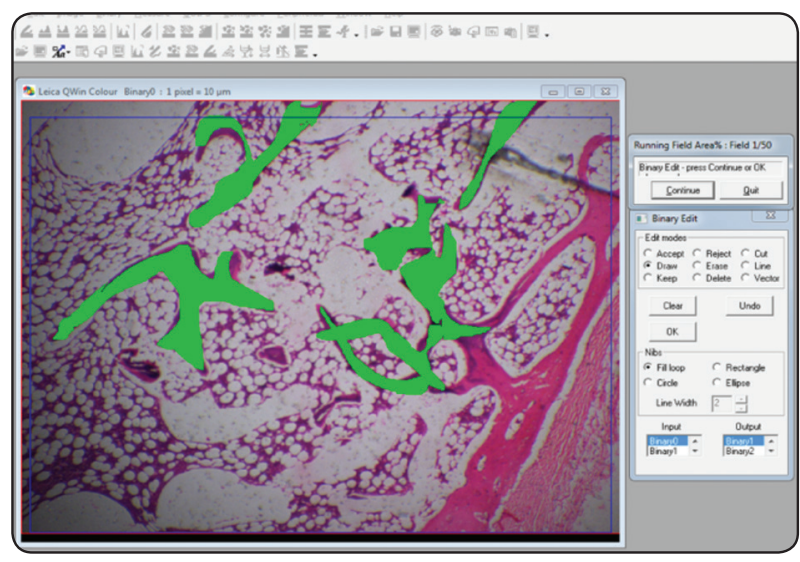

Fig. (4) Steriomicroscope bone density area measurement (100 $\mathrm{X}$ magnification).

\section{Statistical analysis of experimental data:}

The mean and standard deviation (SD) were calculated for all data. Fisher's exact test was used to assess the significance of bacteria clearance among the treatment groups. Differences between the groups were deemed statistically significant if $\mathrm{P}$ value $\leq 0.05$.

\section{RESULTS}

MICs of FOF: The MIC of the used MRSA strain was $0.5 . \mu \mathrm{g} / \mathrm{ml}$.

\section{Bone cultures and bacterial counting:}

Bacterial cultures from bones were positive for MRSA in 8 out of $10(80 \%)$ rabbits in the infected untreated group; mean \pm SD $(2.167 \pm 1.94)$, in 9 out of $10(90 \%)$ rabbits in the fosfomycin-treated group; mean \pm SD $(4.667 \pm 3.386)$ and 9 out of 10 $(90 \%)$ rabbits in the laser treated group Mean \pm SD $(4.000 \pm 2.828)$, the range of bacterial count in the studied group were listed on table (1). No significant difference was found in the bone cultures between the treatment groups and the infected untreated group P value 0.302 .

\section{Radiographic evaluation:}

As regarding radiographic bone density in the three groups we found that highest bone density was in laser group followed by antibiotic treated group and last was the control group but statically there was non- significant difference with p-value was 0.157 (table 1 and figure 5)

Table (1) Bacterial count and bone density among the studied groups

\begin{tabular}{|c|c|c|c|c|c|c|c|c|}
\hline \multirow{2}{*}{ Groups } & \multicolumn{2}{|c|}{ Bacterial count $\mathrm{CFU} / \mathrm{ml}$} & \multicolumn{2}{|c|}{ ANOVA } & \multicolumn{2}{|c|}{ Bone density } & \multicolumn{2}{|c|}{ ANOVA } \\
\hline & Range & Mean \pm SD & $\mathrm{F}$ & $P$ value & Range & Mean \pm SD & $\mathrm{F}$ & $P$ value \\
\hline Control group & $0-5$ & $2.167 \pm 1.94$ & \multirow{3}{*}{1.298} & \multirow{3}{*}{0.302} & $58.8-169.8$ & $128.727 \pm 40.883$ & \multirow{3}{*}{2.097} & \multirow{3}{*}{0.157} \\
\hline Antibiotics group & $0-9$ & $4.667 \pm 3.386$ & & & 114.6-171.3 & $147.818 \pm 26.132$ & & \\
\hline Laser group & $0-7$ & $4.000 \pm 2.828$ & & & $124.02-193.9$ & $165.902 \pm 24.739$ & & \\
\hline
\end{tabular}




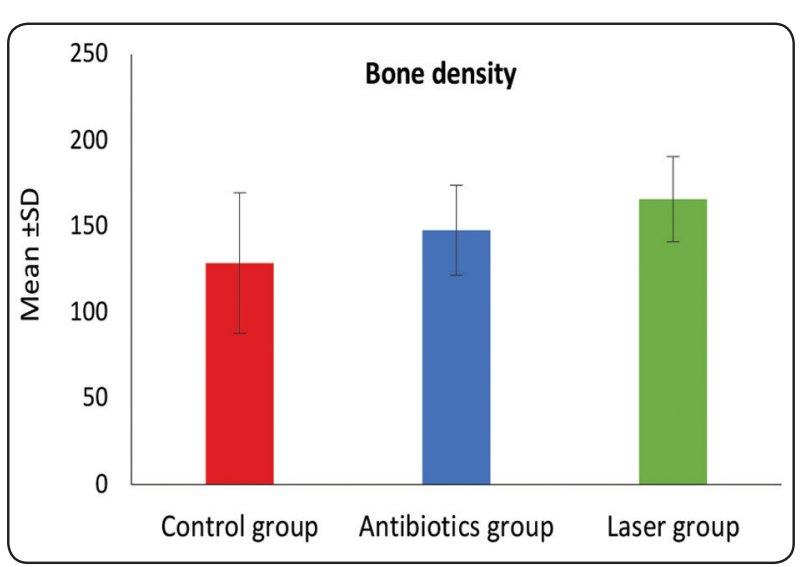

Fig. (5) Bone density among the three studied groups

\section{Correlations between bone density and bacterial count results :}

Correlations between bone density and bacterial count showed no significant differences between groups except in control group there was significant correlation with $\mathrm{p}$ value 0.032 (table 2).

TABLE (2) Correlation between bacterial counts and relative bone density in the studied groups

\begin{tabular}{|c|c|c|}
\hline \multicolumn{3}{|c|}{ Correlations } \\
\hline \multirow{2}{*}{ Bacterial count } & \multicolumn{2}{|c|}{ Bone density } \\
\cline { 2 - 3 } & R & P-value \\
\hline Control group & -0.851 & $0.032^{*}$ \\
\hline Antibiotics group & 0.168 & 0.750 \\
\hline Laser group & -0.441 & 0.381 \\
\hline
\end{tabular}

TABLE (3) Area \% among the three studied groups

\section{Histopathological examination}

The range of area \% and mean SD of the three tested groups were listed in table 3 . There was significant difference between control and laser groups and antibiotic group and laser group with p value $<0.001$ (Figure 5)

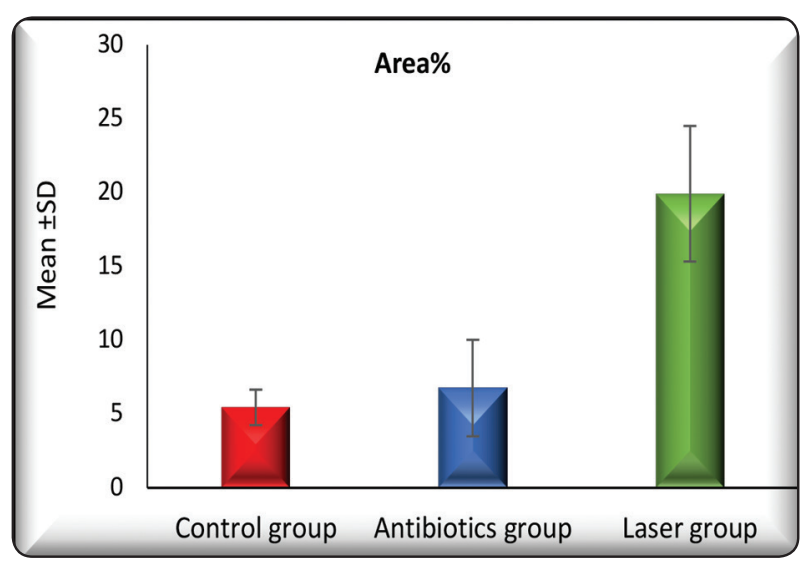

Fig. (6) Area \% of the three studied groups

\section{Histopathological Examination}

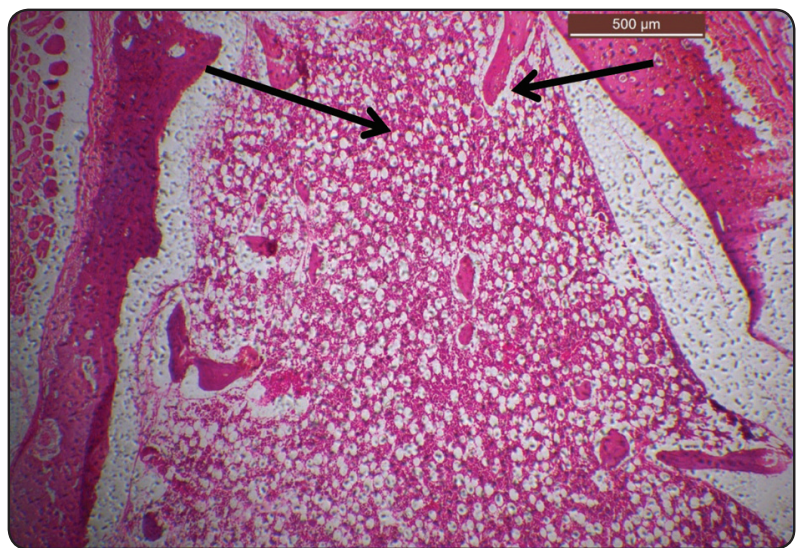

Fig (7): photomicrograph of laser treated group showing proliferation of hemopoietic cells in bone marrow including immunocompetent cells (lymphocytes) (arrows) indicating the activity of cells against the invading bacteria. $\mathrm{H} \& \mathrm{E}$ x 40 .

\begin{tabular}{|c|c|c|c|c|c|c|c|c|}
\hline \multirow{2}{*}{ Groups } & \multicolumn{6}{|c|}{ Area \% } & \multicolumn{2}{|c|}{ ANOVA } \\
\hline & \multicolumn{3}{|c|}{ Range } & Mean & \pm & SD & $\mathbf{F}$ & P-value \\
\hline Control group & 3.84 & - & 7.13 & 5.450 & \pm & 1.201 & \multirow{3}{*}{28.948} & \multirow{3}{*}{$<0.001 *$} \\
\hline Antibiotic group & 3.73 & - & 11.95 & 6.762 & \pm & 3.267 & & \\
\hline Laser group & 13.03 & - & 25.05 & 19.922 & \pm & 4.591 & & \\
\hline \multicolumn{9}{|c|}{ TUKEY'S Test } \\
\hline \multicolumn{2}{|c|}{ Control \& Antibiotics } & & \multicolumn{3}{|c|}{ Control \& Laser } & \multicolumn{3}{|c|}{ Antibiotics \& Laser } \\
\hline \multicolumn{2}{|c|}{0.810} & & \multicolumn{3}{|c|}{$<0.001 *$} & \multicolumn{3}{|c|}{$<0.001^{*}$} \\
\hline
\end{tabular}




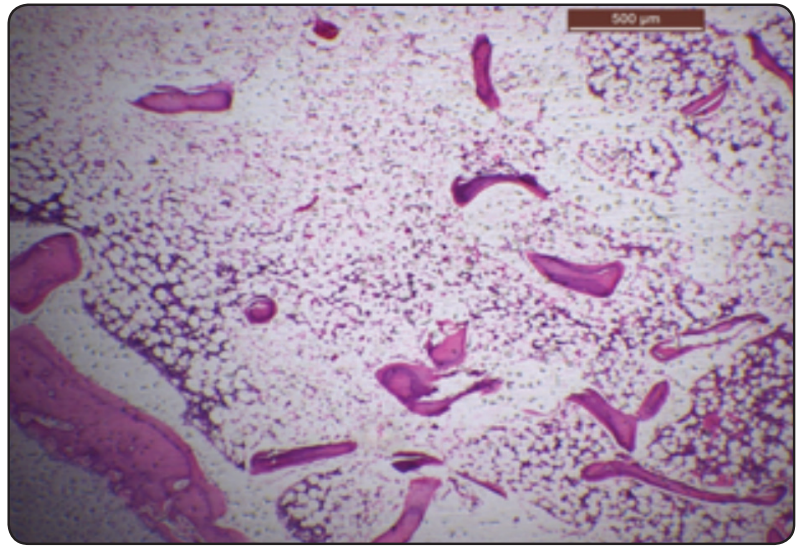

Fig. (8): photomicrograph of Antibiotic treated group showing low proliferation rate of hemopiotic and lymphocytic cells (arrows) that indicated by high number of fat cells (arrow head) compared to laser treated group. H\&E $\mathrm{x} 40$.

\section{DISCUSSION}

Osteomyelitis poses a therapeutic challenge to clinicians especially with the increasing prevalence of MRSA and recent recognition of MRSA with reduced susceptibility to vancomycin (Deresinski,2005). FOF is a bactericidal antibiotic agent, It inhibits the formation of the peptidoglycan precursor in the first cytoplasmic step of the synthesis of the bacterial cell wall (Borisova et al., 2014). FOF has unique pharmacological characteristics and penetrates well into osseous tissue (Schintler et al., 2009).

Fosfomycin was shown to penetrate inside the cells and assist in bacterial clearance in cell line experiments (Valour et al.,2015). In the present study, fosfomycin monotherapy at $75 \mathrm{mg} / \mathrm{kg}$ once daily showed no differences between the three studied groups. However, Poeppl et al., 2011 b showed that FOF at the same dose was effective in the treatment of Methicillin-Resistant Staphylococcus Aureus osteomyelitis in an experimental rat model as it sterilized the bone of 9 rats out of 10 .

Also Poeppl et al., 2011a have previously shown that FOF monotherapy is highly effective

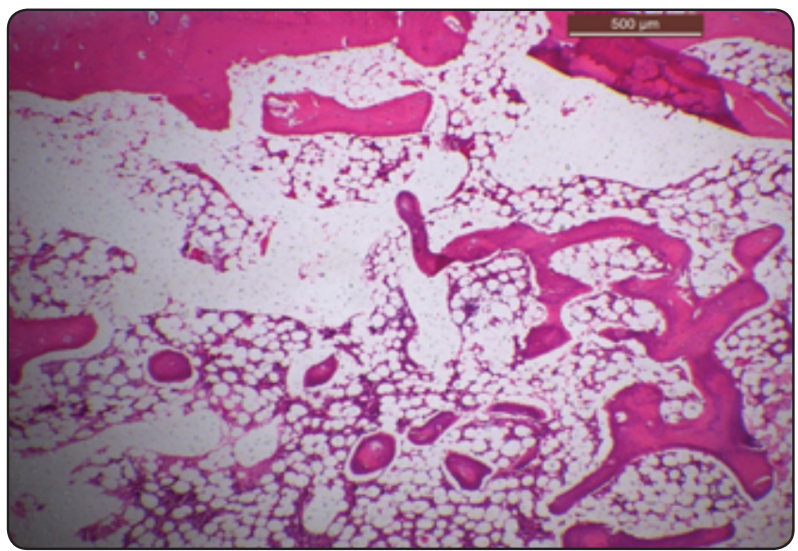

Fig. (9): photomicrograph of control group showing proliferation of hemopiotic and lymphatic cells (arrow) but less than laser group. Fat cells (arrow head) noted but less than antibiotic treated group. H\&E X100.

in experimental MRSA osteomyelitis when administered at a dosage of $150 \mathrm{mg} / \mathrm{kg}$, FOF eradicated MRSA in seven out of nine rats.

It was demonstrated that FOF was effective in the treatment of experimental implant-associated MRSA osteomyelitis when used as a monotherapy (Poeppl et al., 2014). Compared to other antimicrobials, Fosfomycin monotherpy at $40 \mathrm{mg} /$ $\mathrm{Kg}$ of body weight once daily was more active than daptomycin in the treatment of experimental implant-associated osteomyelitis caused by MRSA in rats (Lingscheid et al., 2015). Also Fosfomycin was superior to vancomycin in the treatment of implant-associated chronic MRSA Osteomyelitis in rats(Poeppl et al.,2014).

FOF is used mainly in combination with other classes of antibiotics because of the synergism frequently observed and the concern about the development of resistance (Falagas et al.,2008) .An in vitro study demonstrated enhanced antibacterial activity of linezolid, minocycline, vancomycin, and teicoplanin against isolates of MRSA after the addition of fosfomycin (Tang et al., 2012) however the combination of FOF and daptomycin was not superior to FOF alone (Poeppl et al., 2011b) 
Laser therapy is a low cost, non-invasive procedure with good healing results (Saliva etal., 2013), as Harries and Reinisch-2016 said in their study. The laser mode of antisepsis was currently an uncontrolled consequence of many dental laser procedures. With proper dosimetry it can be developed for specific applications. Selective photoantisepsis may have several potential advantages over traditional antibiotics, a therapeutic dose can be delivered to a greater depth immediately and leaves no residual concentration in the host or environment as an adverse effect was found from uses of antibiotic .

Laser radiation affects equally extracellular and intracellular pathogens and can access other privileged sites and interactions with other modes of therapy. The results of this technique of selective photoantisepsis for killing of MERSA is corresponding to our results. (Kluger et al., 2004)

Kaya et al., 2011 stated that in their study the histopathological analysis showed that infection levels had decreased by using laser therapy. They reported it as a promising for adjunct and/or alternative technique to pharmacological agents in the treatment of osteomyelitis . These results are matching with the results in this study.

\section{CONCLUSION}

Diode Laser $940 \mathrm{~nm} \pm 10 \mathrm{~nm}$ with a dose 4 watt 480 joule for 120 seconds to induce biomodulation effect for two weeks 3 times in each week at site of induced infection of rabbits infected with Staphylococcus aureus MRSA, was shown to reduce bacterial proliferation. It was found to be a good alternative for antibiotics in treatment of osteomyelitis and to have potent antimicrobial activity against MRSA including those with reduced susceptibility to glycopeptides .

\section{REFERENCES}

1. Maynor, M. L., Moon, R. E., Camporesi, E. M., Fawcett, T. A., Fracica, P. J., Norvell, H. C., and Levin, L. S. (1998). Chronic osteomyelitis of the tibia: treatment with hyperbaric oxygen and autogenous microsurgical muscle transplantation. Journal of the Southern Orthopaedic Association, 7(1), 43-57.

2. Waldvogel, F. A., Medoff, G., and Swartz, M. N. (1970) Osteomyelitis: a review of clinical features, therapeutic considerations and unusual aspects. New England Journal of Medicine, 282(5), 260-266.

3. Brady,R.A.,Leid,J.G., Costerton,J.W., and Shirtliff,M.E. (2006). Osteomyelitis: clinical overview and mechanisms of infection persistence. Clinical Microbiology Newsletter, 28(9), 65-72.

4. Conterno, L.O., and Turchi, M.D.(2013): Antibiotics for treating chronic osteomyelitis in adults. Cochrane Database Syst Rev ;9:CD004439.

5. Howden, B. P., Davies, J. K., Johnson, P. D., Stinear, T. P., and Grayson, M. L. (2010). Reduced vancomycin susceptibility in Staphylococcus aureus, including vancomycin-intermediate and heterogeneous vancomycinintermediate strains: resistance mechanisms, laboratory detection, and clinical implications. Clinical microbiology reviews, 23(1), 99-139.

6. Amatya, R., Devkota, P., and Gautam, A. (2014). Reduced susceptibilityto Vancomycin in methicillin resistant Staphylococccusaureus: a time for action. Nepal Med Coll J, 16(1), 42-44.

7. Chen, S. Y., Liao, C. H., Wang, J. L., Chiang, W. C., Lai, M. S., Chie, W. C., ... and Hsueh, P. R. (2013). Methodspecific performance of vancomycin MIC susceptibility tests in predicting mortality of patients with methicillinresistant Staphylococcus aureus bacteraemia. Journal of Antimicrobial Chemotherapy, 69(1), 211-218

8. Tang, H. J., Chen, C. C., Cheng, K. C., Wu, K. Y., Lin, Y. C., Zhang, C. C., \& Chiang, S. R. (2013). In Vitro Efficacy and Resistance of Rifampicin-Based Combination Regimens for Biofilm-Embedded Methicillin-Resistant Staphylococcus aureus. Antimicrobial agents and chemotherapy, AAC-01236.

9. Falagas, M. E., Giannopoulou, K.P., Kokolakis, G.N., and Rafailidis, P.I (2008). Fosfomycin: use beyond urinary tract and gastrointestinal infections. Clin. Infect. Dis. 46:1069-1077 
10. Popovic, M., Steinort, D., Pillai, S., and Joukhadar, C. (2010). Fosfomycin: an old, new friend? Eur. J. Clin. Microbiol. Infect. Dis. 29:127-142

11. Roussos, N., Karageorgopoulos, D. E., Samonis, G., and Falagas, M. E. (2009). Clinical significance of the pharmacokinetic and pharmacodynamic characteristics of fosfomycin for the treatment of patients with systemic infections. International journal of antimicrobial agents, 34(6), 506-515.

12. Clinical and Laboratory Standards Institute. (2014). Performance standards for antimicrobial susceptibility testing, 24th informational supplement. Approved standard M100-S24. Clinical and Laboratory Standards Institute, Wayne, PA.

13. The European Committee on Antimicrobial Susceptibility Testing. (2013):Fosfomycin: rationale for the EUCAST clinical breakpoints, version 1.0

14. Thomas GM, Ashima V, George AI, Denny JP. Laser in Dentistry, Review Article ; Current science. 1993;64:221-223.
15. 15-Kaya GŞ1, Kaya M, Gürsan N, Kireççi E, Güngörmüş $\mathrm{M}$ and Balta $\mathrm{H}$ : The use of 808-nm light therapy to treat experimental chronicosteomyelitis induced in rats by methicillin-resistant Staphylococcus aureus ; Photomed Laser Surg. 2011 Jun; 29(6):405-12. doi: 10.1089/ pho.2010.2807. Epub 2011 Jan 10.

16. 16 - Walaa s, Mushira M D , Mohamed A H, Gihan O and Amal H ; Effect of Various Daily Consumption Agents on Tooth Extraction Wound Healing: Radiographic and Histological Experimental Study, Journal of American Science, 2011;7(12)

17. Harris D M and Reinisch L: Selective Photoantisepsis; JOURNAL OF Lasers in Surgery and Medicine 48:763773 (2016) .

18. Wolf Kluger,Andreas Moritz,Natascha Nedjelik, Apostolos Georgopoulos, and Wolfgang Sperr : Bactericidal Effect of Different Laser Systems in the Deep Layers of Dentin . Lasers in Surgery and Medicine 35:111-116 (2004) 